\title{
The modified equipartition calculation for supernova remnants with the spectral index $\alpha=0.5$
}

\author{
Dejan Urošević, Marko Z. Pavlović, Bojan Arbutina \\ and Aleksandra Dobardžić
}

Department of Astronomy, Faculty of Mathematics, University of Belgrade

Studentski trg 16, 11000 Belgrade, Serbia

email: dejanu@matf.bg.ac.rs

\begin{abstract}
Recently, the modified equipartition calculation for supernova remnants (SNRs) has been derived by Arbutina et al. (2012). Their formulae can be used for SNRs with the spectral indices between $0.5<\alpha<1$. Here, by using approximately the same analytical method, we derive the equipartition formulae useful for SNRs with spectral index $\alpha=0.5$. These formulae represent next step upgrade of Arbutina et al. (2012) derivation, because among 30 Galactic SNRs with available observational parameters for the equipartition calculation, 16 have spectral index $\alpha=0.5$. For these 16 Galactic SNRs we calculated the magnetic field strengths which are approximately 40 per cent higher than those calculated by using Pacholczyk (1970) equipartition and similar to those calculated by using Beck \& Krause (2005) calculation.
\end{abstract}

Keywords. ISM: magnetic fields, ISM: supernova remnants, radio continuum: general

This paper represents next step upgrade of Arbutina et al. (2012) determination of the magnetic field strength in the interstellar medium. We obtained analytical formula for calculation of the magnetic field for SNRs with the radio spectral index $\alpha=0.5$ :

$$
B[\mathrm{G}]=9.1 \cdot 10^{-5}\left[\left(1+\kappa_{\gamma=2}\right) \frac{\mathrm{S}_{\nu}[\mathrm{Jy}]}{\mathrm{f} \theta[\operatorname{arcmin}]^{3} \mathrm{~d}[\mathrm{kpc}]}\right]^{2 / 7} \nu[\mathrm{GHz}]^{1 / 7}
$$

Our values for SNRs with $\alpha=0.5$ are slightly lower than those obtained by applying Beck \& Krause (2005) revised equipartition formula for $\alpha=0.5$. Applying our formula yields to magnetic field strengths which are approximately $40 \%$ higher than those calculated by using classical approach derived by Pacholczyk (1970). This is mostly because we were using a wider interval of integration than standard Pacholczyk interval of 10 $\mathrm{MHz}-10 \mathrm{GHz}$.

The Web application for calculation of the magnetic field strengths of SNRs for the spectral indices $0.5<\alpha<1$ is available at http://poincare.matf.bg.ac.rs/ arbo/ eqp/ and value $\alpha=0.578$ should be used instead of $\alpha=0.5$.

During the work on this paper, the authors were financially supported by the Ministry of Education and Science of the Republic of Serbia through the project number 176005 .

\section{References}

Arbutina, B., Urošević, D., Andjelić, M. M., Pavlović, M. Z., \& Vukotić, B. 2012, ApJ, 746, 79 Beck, R. \& Krause, M. 2005, Astron. Nachr., 326, 414

Pacholczyk, A. G. 1970, Radio Astrophysics (San Francisco, CA:Freeman) 